\title{
Increasing the User Experience in Autonomous Driving through different Feedback Modalities
}

\author{
Tobias Schneider \\ Stuttgart Media University \\ Stuttgart, Germany \\ schneidert@hdm-stuttgart.de \\ Steve Love \\ The Glasgow School of Art \\ Glasgow, Schotland \\ s.love@gsa.ac.uk
}

\author{
Sabiha Ghellal \\ Stuttgart Media University \\ Stuttgart, Germany \\ ghellal@hdm-stuttgart.de \\ Ansgar Gerlicher \\ Stuttgart Media University \\ Stuttgart, Germany \\ gerlicher@hdm-stuttgart.de
}

\begin{abstract}
Within the ongoing process of defining autonomous driving solutions, experience design may represent an important interface between humans and the autonomous vehicle. This paper presents an empirical study that uses different ways of unimodal communication in autonomous driving to communicate awareness and intent of autonomous vehicles. The goal is to provide recommendations for feedback solutions within holistic autonomous driving experiences. 22 test subjects took part in four autonomous, simulated virtual reality shuttle rides and were presented with different unimodal feedback in the form of light, sound, visualisation, text and vibration. The empirical study showed that, compared to a nofeedback baseline ride, light, and visualisation were able to create a positive user experience.
\end{abstract}

\section{CCS CONCEPTS}

- Human-centered computing $\rightarrow$ Empirical studies in $\mathrm{HCI}$; User centered design; Interaction techniques; Interaction devices;

\section{KEYWORDS}

user experience, explainable artificial intelligence, autonomous driving

\section{ACM Reference Format:}

Tobias Schneider, Sabiha Ghellal, Steve Love, and Ansgar Gerlicher. 2021. Increasing the User Experience in Autonomous Driving through different Feedback Modalities. In 26th International Conference on Intelligent User Interfaces (IUI '21), April 14-17, 2021, College Station, TX, USA. ACM, New York, NY, USA, 4 pages. https://doi.org/10.1145/3397481.3450687

\section{INTRODUCTION}

With vehicle assistant systems being on the rise for multiple years, autonomous vehicles (AVs) are starting to become reality $[3,7,28$, $32,35]$. Previous studies proof that losing the possibility of control may lead to a negative User Experience (UX), especially in urban

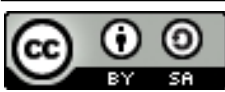

This work is licensed under a Creative Commons Attribution-ShareAlike International 4.0 License.

IUI '21, April 14-17, 2021, College Station, TX, USA

(C) 2021 Copyright held by the owner/author(s)

ACM ISBN 978-1-4503-8017-1/21/04

https://doi.org/10.1145/3397481.3450687 areas with a lot of situations happening at the same time [11, 17, 36]. Providing appropriate feedback could address potential issues and lead to more positive experiences. We argue that autonomous driving immersion via feedback modalities should play a major role in the holistic User Experience Design (UXD) of an automotive vehicle and that by communication awareness and intent via unimodal feedback modalities passengers can have a better understanding of the AV's actions and an improved overall UX. In this paper, we introduce and discuss autonomous driving immersion focusing on light, audio, text, visualisation and vibration feedback.

\section{RELATED WORK}

Making artificial systems understandable to humans is part of the explainable artificial intelligence (XAI) research area $[1,8,23]$ and focuses on explainability and explanation [21]. One problem is that experts in the field of artificial intelligence (AI) may not always be the right ones to explain these complex models [8,21, 22, 31]. Therefore, simpler, context-dependent and well-timed human-friendly ways of communication have to be found $[13,21]$. The connection of UXD and XAI and the possible increase in transparency in the human-AI interaction motivate this paper. Using single (unimodal) or multiple (multimodal) feedback modalities to communicate awareness and intent in (semi-)autonomous driving has been the topic of research in recent years. Multiple studies have shown the usefulness of feedback modalities such as light, audio, visualisation, text or vibration and their combinations to convey information to a driver or passenger [12, 16, 18, 19, 24-27, 34]. They can increase the understanding of a machine's decision, and its limits $[4,10,20,30,37]$ as well as help to increase the feeling of trust and safety of a passenger $[9,15]$. However, none of these studies have purely focused on the UX in autonomous driving when it comes to communicating the AI's awareness and intent. Therefore, this paper wants to provide a first step in this direction.

\section{PROTOTYPE}

\subsection{Driving Situations}

In the context of autonomous driving, different types of situations can occur. During a workshop with researchers and automotive employees at a research campus, the following four different categories of driving situations were identified: proactive and reactive ones, which both can either be critical or non-critical. 

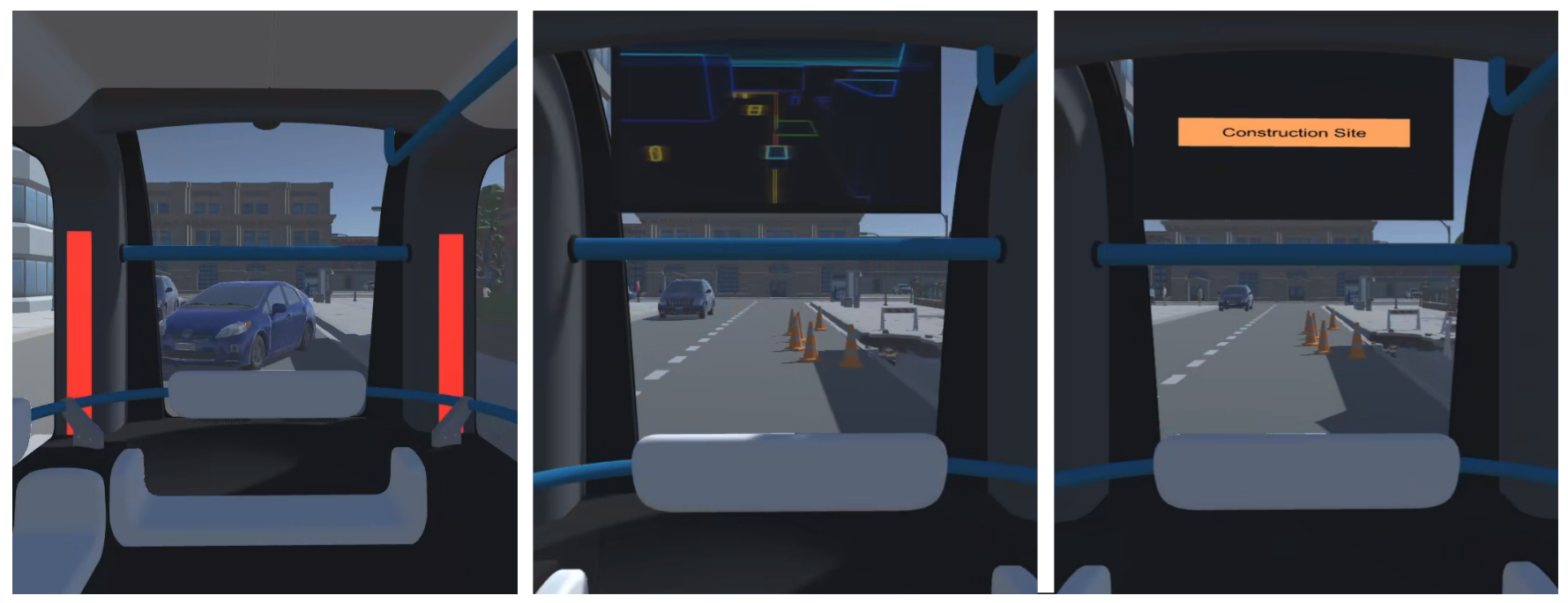

Figure 1: Three out of the five designed modalities. Left: reactive critical - light, middle: proactive non-critical - visualisation, right: proactive non-critical - text.

Proactive Non-Critical: The AV has sufficient reaction time for a situation that does not endanger human health or lives. For example, a small construction site is in the vehicle's way and needs to be bypassed. The AV has to wait until oncoming traffic passed and can then bypass the construction site.

Proactive Critical: The AV has sufficient reaction time for a situation that does endanger human health or lives. For example, a car in oncoming traffic is overtaking the car in front. The AV recognises the situation and brakes early, to avoid a dangerous situation.

Reactive Non-Critical: The AV has insufficient reaction time for a situation that does not endanger human health or lives. For example, a dog suddenly appears behind a parked car on the side and runs across the street. The AV has to perform an emergency brake.

Reactive Critical: The AV has insufficient reaction time for a situation that does endanger human health or lives. For example, a car in oncoming traffic is overtaking the car in front. The AV has to perform an emergency brake to avoid an accident.

\subsection{Feedback Design}

A virtual reality (VR) prototype was created in Unity [33]. Following the aforementioned studies' research, a set of five different feedback modalities was designed: light, visualisation, text, audio and vibration, which all offer different depths of information.

Light: Based on the related work, light bars in the front left and right of the vehicle use a sort of traffic light system to indicate driving status, and reactions [20,30]. White: everything is normal, green: an obstacle was recognised, but a reaction is not necessary, yellow: an obstacle was recognised, and the vehicle will brake, red: risk of collision was recognised, the vehicle will perform an emergency braking. If events happen at the front left of the vehicle, the left light bar changes colour. The same applies to the right side.
If something happens directly in front of the vehicle, both light bars change colour.

Audio: Passengers were presented with abstract sounds for three different types of situations, so-called auditory icons or earcons [5]. Continue drive: a short, relaxing sounding two-note chime signalling that the shuttle continues its drive. Reaction: a neutral sounding two-note chime signalling that a situation was recognised and the shuttle will react. Warning: three consecutive beep sounds that sound alerting and communicate dangerous situations.

Visualisation: A visualisation, inspired by the world of a miniature representation of [15] and Tesla and Waymo [32,35] is shown to the passengers via a display. It highlights recognised objects, vehicles and pedestrians as well as route information.

Text: When the vehicle performs actions related to driving situations, they are displayed via a short text. The focus lies on whymessages, as introduced in [18], where testers reported a better driving performance. For the four different driving situations in the prototype it says "construction site", "overtaking", "!!animal!!" and "!!ghost driver!!". Visually, it is always displayed the same way.

Vibration: Vibration patterns in the seat are used to communicate different types of events as in [16, 24, 25]. Proactive situation: vibrating three times with a duration of 0.3 seconds per vibration. Reactive situation: vibrating six times with a shorter duration of 0.1 seconds per vibration.

\subsection{Driving Simulator}

For each of the four aforementioned driving situations, a virtual driving scene was created, taking place in a single-lane urban area. The AV was driving at around $30 \mathrm{~km} / \mathrm{h}$, and all driving situations took around 25 seconds to experience. Besides the vibration, all feedback modalities are part of the virtual world. The vibration was realised via a vibration motor that is connected to an Arduino Uno [2] which communicated directly with the Unity prototype. The motor was installed in a small metal tube which lied on the sitting surface. 


\begin{tabular}{lcccccc}
\hline & No Feedback & Light & Sound & Visualisation & Text & Vibration \\
\hline Pragmatic & $\mathbf{0 . 5 5}$ & $\mathbf{2 . 0 5}$ & $\mathbf{1 . 1 9}$ & $\mathbf{1 . 1 5}$ & $\mathbf{0 . 8 5}$ & $\mathbf{0 . 0 1}$ \\
Quality & $\alpha=0.77$ & $\alpha=0.94$ & $\alpha=0.88$ & $\alpha=0.94$ & $\alpha=0.82$ & $\alpha=0.79$ \\
\hline Hedonic & $\mathbf{- 0 . 4 0}$ & $\mathbf{0 . 6 3}$ & $\mathbf{- 0 . 3 5}$ & $\mathbf{1 . 6 0}$ & $\mathbf{- 0 . 4 1}$ & $\mathbf{0 . 6 3}$ \\
Quality & $\alpha=0.86$ & $\alpha=0.91$ & $\alpha=0.93$ & $\alpha=0.63$ & $\alpha=0.85$ & $\alpha=0.87$ \\
\hline \multirow{2}{*}{ Overall } & $\mathbf{0 . 0 7}$ & $\mathbf{1 . 3 4}$ & $\mathbf{0 . 4 2}$ & $\mathbf{1 . 3 8}$ & $\mathbf{0 . 2 2}$ & $\mathbf{0 . 3 2}$ \\
& & $\mathrm{p}<.001$ & $\mathrm{p}=.171$ & $\mathrm{p}<.001$ & $\mathrm{p}=.864$ & $\mathrm{p}=.442$
\end{tabular}

Table 1: UEQ-S results of the different feedback modalities. The p-values refer to the no-feedback baseline comparison.

\section{STUDY DESIGN}

Based on related work, we present the following hypothesis:

Hypothesis $\mathbf{H}_{\mathbf{1}}$ : Different, single feedback modalities in the form of light, audio, visualisation, text and vibration will create a positive passenger UX in different driving situations.

A mixed-method within-subjects design was used for this experiment. All testers experienced all four driving situations with all feedback modalities, which were counterbalanced with a Latin square design. All subjects were first-time users with no prior information about the different modalities. The UX, in the form of pragmatic and hedonic quality [14], was measured using the UEQ-S [29]. Testers were asked to answer the questionnaire regarding the feedback they experienced during the rides. For the baseline ride without any feedback, they were told to only rate the shuttle's driving actions as feedback. Qualitative feedback was collected during and after the tests via the think-aloud method and an unstructured interview. Afterwards, testers were asked to state which feedback modality they liked best in which situation and which pair of feedback modalities they would like to have in each situation.

The 22 participants $(12$ female, medium age $=28.36, \mathrm{SD}=4.04$ ) did not share a common background and were recruited from a university (students and employees), different research institutes and the industry. All of them had a valid driver's license and no prior experience with fully autonomous driving. All reported normal or corrected-to-normal vision and no physical constraints. Since all of the testers were first-time users, they were introduced to the prototype via a VR tutorial which explained the different feedback modalities. They did not experience any distractions or a secondary task during the different drives. Each test took roughly one hour.

\section{RESULTS AND DISCUSSION}

\subsection{User Experience Questionnaire - Short}

Table 1 shows the results for the different feedback rides. Reliability was measured using Cronbach alpha [6]. The UEQ-S states values > 0.8 as a positive evaluation and values $<-0.8$ as a negative evaluation. Since the UEQ-S data was not normally distributed a Friedman's Two-Way Analysis was performed.

Looking at the results, there is an indication that the hypothesis $\mathrm{H}_{1}$ is partly valid. For every feedback modality, the UEQ-S scores were higher than the no-feedback baseline. However, not all feedback modalities created a significantly better UX. The feedback modalities that showed only neutral overall scores were sound, text and vibration. Sound had a positive pragmatic quality (PQ) but a negative neutral hedonic quality (HQ). This is consistent with the testers' statements, who said that the sound feedback might be annoying in the long run (10 mentions). The feedback modality text scored the worst overall score of all feedback modalities. It was described as redundant (6), was missing some sort of colour highlight (10) and took testers too long to read and process (6). Vibration also had a low overall score and is the only feedback modality with a lower PQ than the no-feedback baseline. The results are supported by testers saying that vibration is annoying and unpleasant (11), not always understandable (3) and creates a feeling that one needs to act, which they cannot as passive passengers (4).

The feedback modalities light and visualisation both created a significantly positive UX compared to the baseline $(\mathrm{p}<.001)$. The light's high positive PQ is supported by it being useful and well understandable in some or all situations (18). However, it only scored a neutral HQ which might be due to too many gradients of light, which felt unnecessary and provided too much feedback (12). The feedback modality visualisation is the only one that scored positively for both $\mathrm{PQ}$ and $\mathrm{HQ}$ and achieved the best overall score of all modalities. Its positive PQ is supported by the testers' statements that it is helpful (7). Highlighting relevant things in the visualisation (7) might increase its PQ even more. The high HQ is supported by testers stating that it is reassuring to see what the AV sees (8).

\subsection{Favourite Feedback Modalities}

Testers preferred softer, less intrusive feedback for the proactive situations (light or visualisation) and more prominent feedback for the reactive ones (sound or light), which is consistent with what Politis et al. found out [26]. Looking at the desired feedback modality pairs, testers wished for the pairs light \& visualisation and visualisation \& text for the proactive situations. This is supported by their think-aloud statements, saying that visualisation is missing an intention (6) and should be combined with text (3). For the reactive situations, more prominent feedback was preferred again, with light being either extended by sound or vibration.

\section{LIMITATIONS}

As with all empirical studies, there are certain limitations to the experiment. While VR is an immersive medium and situations like the critical overtaking scared testers, which showed that there is an emotional reaction to the situations, a digital world is always only a representation of the real world and may lack immersion due to graphic, sound or g-forces limitations. Moreover, testers were told to only focus on the ride to achieve comparability between the different feedback modalities. In a real-world scenario, passengers of an AV might be working, listening to music or having a conversation 
and might not focus on the ride as much. Furthermore, all testers experienced the different modalities for the first time and only for four rides. Also, only people with a median age of 28 were tested.

\section{CONCLUSION \& OUTLOOK}

The study shows that, overall, the different feedback modalities were able to create a better UX compared to the no-feedback baseline. However, only light and visualisation were able to create a significantly positive UX. For the different driving situations, testers preferred softer, less intrusive feedback modalities for proactive situations and more prominent feedback for the reactive ones. This is true for unimodal feedback and feedback pairs and shows that it might be reasonable to present an AV's driving decision with different feedback modalities depending on the situation. Further studies will take a look at the combination of light and visualisation and their impact on the understanding of the actions of an AV as well as their implications on the UX of an autonomous ride.

\section{ACKNOWLEDGMENTS}

This research was carried out as part of the FlexCAR project of the Arena2036 research campus and was funded by the German Federal Ministry of Education and Research (funding number: 02P18Q647).

\section{REFERENCES}

[1] Amina Adadi and Mohammed Berrada. 2018. Peeking Inside the Black-Box: A Survey on Explainable Artificial Intelligence (XAI). IEEE Access 6 (2018), 5213852160. https://doi.org/10.1109/ACCESS.2018.2870052

[2] Arduino. 2020. Arduino Uno Rev3. Retrieved January, 2020 from https://store. arduino.cc/arduino-uno-rev3

[3] Audi. 2020. Autonomous Driving. Retrieved January, 2020 from https://www. audi.com/en/experience-audi/mobility-and-trends/autonomous-driving.html

[4] Johannes Beller, Matthias Heesen, and Mark Vollrath. 2013. Improving the DriverAutomation Interaction - An Approach Using Automation Uncertainty. Human Factors 55, 6 (2013), 1130-1141.

[5] Stephen Brewster, Peter C Wright, and Alistair D N Edwards. 1993. An evaluation of earcons for use in auditory human-computer interfaces. In the SIGCHI conference. ACM Press, New York, New York, USA, 222-227.

[6] Lee J Cronbach. 1951. Coefficient alpha and the internal structure of tests. Psychometrika 16, 3 (Sept. 1951), 297-334.

[7] Daimler AG. 2020. Autonomous Driving. Retrieved January, 2020 from https: //www.daimler.com/innovation/product-innovation/autonomous-driving/

[8] Filip Karlo Dosilovic, Mario Brcic, and Nikica Hlupic. 2018. Explainable artificial intelligence - A survey.. In 2018 41st International convention on information and communication technology, electronics and microelectronics (MIPRO). IEEE, 0210-0215.

[9] Fredrick Ekman, Mikael Johansson, and Jana Sochor. 2016. To See or Not to See - The Effect of Object Recognition on Users' Trust in "Automated Vehicles".. In Proceedings of the 9th Nordic Conference on Human-Computer Interaction. ACM Press, New York, New York, USA, 1-4.

[10] Sarah Faltaous, Martin Baumann, Stefan Schneegass, and Lewis Chuang. 2018. Design Guidelines for Reliability Communication in Autonomous Vehicles.. In Proceedings of the 7th international conference on automotive user interfaces and interactive vehicular applications. ACM Press, New York, New York, USA, 258267.

[11] Anna-Katharina Frison, Philipp Wintersberger, Tianjia Liu, and Andreas Riener 2019. Why do you like to drive automated? - a context-dependent analysis of highly automated driving to elaborate requirements for intelligent user interfaces. In Proceedings of the 24th International Conference on Intelligent User Interfaces. ACM Press, New York, New York, USA, 528-537.

[12] Nick Gang, Srinath Sibi, Romain Michon, Brian K Mok, Chris Chafe, and Wendy Ju. 2018. Don't Be Alarmed - Sonifying Autonomous Vehicle Perception to Increase Situation Awareness.. In Proceedings of the 7th international conference on automotive user interfaces and interactive vehicular applications. ACM Press, New York, New York, USA, 237-246.

[13] Jacob Haspiel, Na Du, Jill Meyerson, Lionel P Robert Jr, Dawn Tilbury, X Jessie Yang, and Anuj K Pradhan. 2018. Explanations and expectations: Trust building in automated vehicles. In Companion of the 2018 ACM/IEEE International Conference on Human-Robot Interaction. Association for Computing Machinery, 119-120.
[14] Marc Hassenzahl. 2007. The hedonic/pragmatic model of user experience. Towards a UX manifesto 10 (2007).

[15] Renate Häuslschmid, Max von Bülow, Bastian Pfleging, and Andreas Butz. 2017. SupportingTrust in Autonomous Driving. ACM, New York, New York, USA

[16] Cristy Ho, Hong Z Tan, and Charles Spence. 2005. Using spatial vibrotactile cues to direct visual attention in driving scenes. Transportation Research Part F: Traffic Psychology and Behaviour 8, 6 (Nov. 2005), 397-412.

[17] Myounghoon Jeon, Andreas Riener, Jason Sterkenburg, Ju-Hwan Lee, Bruce N Walker, and Ignacio Alvarez. 2018. An International Survey on Automated and Electric Vehicles: Austria, Germany, South Korea, and USA. In Digital Human Modeling. Applications in Health, Safety, Ergonomics, and Risk Management. Springer, Cham, Cham, 579-587.

[18] Jeamin Koo, Jungsuk Kwac, Wendy Ju, Martin Steinert, Larry Leifer, and Clifford Nass. 2014. Why did my car just do that? Explaining semi-autonomous driving actions to improve driver understanding, trust, and performance. International fournal on Interactive Design and Manufacturing (IFIDeM) 9, 4 (April 2014), 269275.

[19] Bridget A Lewis, B N Penaranda, Daniel M Roberts, and Carryl L Baldwin. 2017. Effectiveness of Bimodal Versus Unimodal Alerts for Distracted Drivers. In Driving Assessment Conference. University of Iowa, Iowa City, Iowa, 376-382.

[20] B W Meerbeek, C de Bakker, Y A W de Kort, E J van Loenen, and T Bergman. 2016. Automated blinds with light feedback to increase occupant satisfaction and energy saving. Building and Environment 103 (July 2016), 70-85.

[21] Tim Miller. 2019. Explanation in artificial intelligence: Insights from the social sciences. Artificial Intelligence 267 (Feb. 2019), 1-38.

[22] Tim Miller, Piers Howe, and Liz Sonenberg. 2017. Explainable AI - Beware of Inmates Running the Asylum Or - How I Learnt to Stop Worrying and Love the Social and Behavioural Sciences. arXiv preprint arXiv:1712.00547 (2017).

[23] Sina Mohseni, Niloofar Zarei, and Eric D. Ragan. 2018. A Survey of Evaluation Methods and Measures for Interpretable Machine Learning. CoRR abs/1811.11839 (2018). arXiv:1811.11839 http://arxiv.org/abs/1811.11839

[24] Ioannis Politis, Stephen Brewster, and Frank Pollick. 2014. Evaluating multimodal driver displays under varying situational urgency.. In Ijcai-2016 ethics for artificial intelligence workshop. ACM Press, New York, New York, USA, 4067-4076.

[25] Ioannis Politis, Stephen Brewster, and Frank Pollick. 2015. Language-based multimodal displays for the handover of control in autonomous cars.. In Proceedings of the 7th international conference on automotive user interfaces and interactive vehicular applications. ACM Press, New York, New York, USA, 3-10.

[26] Ioannis Politis, Stephen Brewster, and Frank Pollick. 2015. To Beep or Not to Beep? - Comparing Abstract versus Language-Based Multimodal Driver Displays.. In Ijcai-2016 ethics for artificial intelligence workshop. ACM Press, New York, New York, USA, 3971-3980.

[27] Ioannis Politis, Stephen Brewster, and Frank Pollick. 2017. Using Multimodal Displays to Signify Critical Handovers of Control to Distracted Autonomous Car Drivers. IfMHCI 9, 3 (2017), 1-16.

[28] SAE On-Road Automated Vehicle Standards Committee and others. 2018. Taxonomy and Definitions for Terms Related to Driving Automation Systems for On-Road Motor Vehicles. SAE International Warrendale, PA, USA (2018).

[29] Martin Schrepp, Andreas Hinderks, and Jörg Thomaschewski. 2017. Design and Evaluation of a Short Version of the User Experience Questionnaire (UEQ-S). IfIMAI 4, 6 (2017), 103.

[30] Bobbie D Seppelt and John D Lee. 2007. Making adaptive cruise control (ACC) limits visible. International fournal of Human-Computer Studies 65, 3 (March 2007), 192-205.

[31] Erik Strumbelj and Igor Kononenko 0001. 2014. Explaining prediction models and individual predictions with feature contributions. Knowl. Inf. Syst. 41, 3 (2014), 647-665.

[32] Tesla. 2020. Autopilot. Retrieved January, 2020 from https://www.tesla.com/ autopilot?redirect=no

[33] Unity. 2020. Unity - Unity. Retrieved January, 2020 from https://unity.com/

[34] Marcel Walch, Kristin Lange, Martin Baumann, and Michael Weber. 2015. Autonomous driving - investigating the feasibility of car-driver handover assistance.. In Proceedings of the 7th international conference on automotive user interfaces and interactive vehicular applications. ACM Press, New York, New York, USA, 11-18.

[35] Waymo. 2020. Waymo - Waymo. Retrieved January, 2020 from https://waymo. com

[36] Philipp Wintersberger, Andreas Riener, and Anna-Katharina Frison. 2016. Automated Driving System, Male, or Female Driver: Who'd You Prefer? Comparative Analysis of Passengers' Mental Conditions, Emotional States \& Qualitative Feedback. ACM, New York, New York, USA.

[37] Robert Wortham, Andreas Theodorou, and Joanna Bryson. 2016. What does the robot think? Transparency as a fundamental design requirement for intelligent systems. In Ijcai-2016 ethics for artificial intelligence workshop. 